University of Wollongong

Research Online

Australian Institute for Innovative Materials -

Papers

Australian Institute for Innovative Materials

$1-1-2014$

\title{
Fast and rewritable colloidal assembly via field synchronized particle swapping
}

Pietro Tierno

Universitat De Barcelona

Tom H. Johansen

University of Wollongong, tomjo@uow.edu.au

Thomas M. Fischer

Universitat Bayreuth

Follow this and additional works at: https://ro.uow.edu.au/aiimpapers

Part of the Engineering Commons, and the Physical Sciences and Mathematics Commons

Research Online is the open access institutional repository for the University of Wollongong. For further information contact the UOW Library: research-pubs@uow.edu.au 


\title{
Fast and rewritable colloidal assembly via field synchronized particle swapping
}

\author{
Abstract \\ We report a technique to realize reconfigurable colloidal crystals by using the controlled motion of \\ particle defects above an externally modulated magnetic substrate. The transport of particles is induced \\ by applying a uniform rotating magnetic field to a ferrite garnet film characterized by a periodic lattice of \\ magnetic bubbles. For filling factor larger than one colloid per bubble domain, the particle current arises \\ from propagating defects where particles synchronously exchange their position when passing from one \\ occupied domain to the next. The amplitude of an applied alternating magnetic field can be used to \\ displace the excess particles via a swapping mechanism, or to mobilize the entire colloidal system at a \\ predefined speed.

\section{Keywords} \\ synchronized, rewritable, assembly, fast, via, particle, field, colloidal \\ Disciplines \\ Engineering | Physical Sciences and Mathematics

\section{Publication Details} \\ Tierno, P., Johansen, T. \& Fischer, T. (2014). Fast and rewritable colloidal assembly via field synchronized \\ particle swapping. Applied Physics Letters, 104 (17), 174102-1-174102-5.
}




\title{
Fast and rewritable colloidal assembly via field synchronized particle swapping
}

\author{
Pietro Tierno, ${ }^{1,2, a)}$ Tom H. Johansen, ${ }^{3,4}$ and Thomas M. Fischer ${ }^{5}$ \\ ${ }^{1}$ Departament d'Estructura i Constituents de la Matèria, Universitat de Barcelona, Avinguda Diagonal 647 , \\ 08028 Barcelona, Spain \\ ${ }^{2}$ Institut de Nanociència i Nanotecnologia IN2UB, Universitat de Barcelona, Avinguda Diagonal 647, \\ 08028 Barcelona, Spain \\ ${ }^{3}$ Department of Physics, University of Oslo, P. O. Box 1048, Blindern, Norway \\ ${ }^{4}$ Institute for Superconducting and Electronic Materials, University of Wollongong, Wollongong, NSW 2522, \\ Australia \\ ${ }^{5}$ Institut für Experimentalphysik V, Universität Bayreuth, 95440 Bayreuth, Germany
}

(Received 19 March 2014; accepted 21 April 2014; published online 2 May 2014)

\begin{abstract}
We report a technique to realize reconfigurable colloidal crystals by using the controlled motion of particle defects above an externally modulated magnetic substrate. The transport of particles is induced by applying a uniform rotating magnetic field to a ferrite garnet film characterized by a periodic lattice of magnetic bubbles. For filling factor larger than one colloid per bubble domain, the particle current arises from propagating defects where particles synchronously exchange their position when passing from one occupied domain to the next. The amplitude of an applied alternating magnetic field can be used to displace the excess particles via a swapping mechanism, or to mobilize the entire colloidal system at a predefined speed. (C) 2014 AIP Publishing LLC. [http://dx.doi.org/10.1063/1.4874839]
\end{abstract}

The flow of electrical current in a conductor arises when electrons at the Fermi level are scattered from occupied states into unoccupied ones due to the interaction with an external electric field. Following this electronic analogy, it is a compelling idea to find ways to separate an ensemble of identical colloidal particles into a set of immobile low energy particles, and colloids which can become mobile due to an external field. When applied to microtechnological devices like lab-on-a-chip, this concept has demonstrated precise single particle operations based on the selective motion of colloidal inclusions. ${ }^{1-3}$

Paramagnetic microspheres, which can be remotely controlled via non invasive magnetic fields, are currently employed in biotechnological applications. ${ }^{4-6}$ The surfaces of these particles can be chemically functionalized allowing to bind selectively to defined targets. ${ }^{7}$ For an ensemble of monodisperse particles, the formation of a threshold energy where only a fraction of particles will move in response to an applied magnetic field is difficult to achieve. In most cases, the field-induced interactions between the particles favor collective motion rather than selected displacements. Magnetic patterned substrates have shown considerable potential to overcome the above limitation. These patterns can generate strong localized field gradients, allowing controlled particle trapping and transport along predefined magnetic tracks. Recent experiments include the use of arrays of permalloy elliptical islands, ${ }^{8}$ cobalt microcylinders, ${ }^{9}$ domain wall conduits, ${ }^{10}$ magnetic wires, ${ }^{11}$ exchange bias layer systems, ${ }^{12}$ and magnetic micromoulds. ${ }^{13}$

An alternative method consists in using epitaxially grown ferrite garnet films (FGFs), where magnetic domains with the width of few microns, i.e., on the particle scale, self-assemble into patterns of stripes or bubbles. ${ }^{14}$ Originally developed for

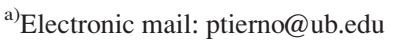

magnetic memory applications ${ }^{15}$ and magneto-optical imaging, ${ }^{16}$ the FGFs are ideal to manipulate paramagnetic colloids, ${ }^{17}$ or superconducting vortices. ${ }^{18}$ The highly localized driving force originates from the stray field generated by the Bloch walls (BWs) in the FGF. When properly synthesized, the displacement of the BWs caused by an external field is smooth and reversible, with absence of wall pinning, thus creating a precise and controllable driving force for the particle motion. Here, we use a FGF to manipulate and transport an ensemble of paramagnetic particles or a fraction of it, demonstrating a technique to dynamically organize a colloidal system into trapped immobile particles and particles which become mobile above a threshold field.

A bismuth-substituted ferrite garnet of composition $\mathrm{Y}_{2.5} \mathrm{Bi}_{0.5} \mathrm{Fe}_{5-q} \mathrm{Ga}_{q} \mathrm{O}_{12}(q=0.5-1)$ was prepared by liquid phase epitaxy on a (111)-oriented gadolinium gallium garnet (GGG) substrate. Oxide powders of the constituent elements, as well as $\mathrm{PbO}$ and $\mathrm{B}_{2} \mathrm{O}_{3}$, were initially melted at $1050{ }^{\circ} \mathrm{C}$ in a platinum crucible, while the GGG wafer was located horizontally just above the melt surface. After lowering the temperature to $700{ }^{\circ} \mathrm{C}$, growth of the FGF was started by letting the substrate touch the melt. Keeping it there for $8 \mathrm{~min}$ produced a FGF of $5 \mu \mathrm{m}$ thickness, more details can be found in a previous work. ${ }^{19}$ At ambient temperature, the FGF has a spontaneous magnetization perpendicular to the plane of the film. To minimize the magnetic energy, the FGF breaks up into domains characterized by a labyrinth stripe pattern, easily observed by polarized light microscopy due to the large Faraday effect in this material. High frequency magnetic fields were used to transform this pattern into a regular triangular lattice of "magnetic bubbles." These are cylindrical ferromagnetic domains magnetized oppositely to the remaining continuous area of the FGF, and separated by BWs. The magnetic bubbles have in zero field a diameter of $6.4 \mu \mathrm{m}$ and a lattice constant of $l=8.6 \mu \mathrm{m}$, Fig. 1(a). 
(a)
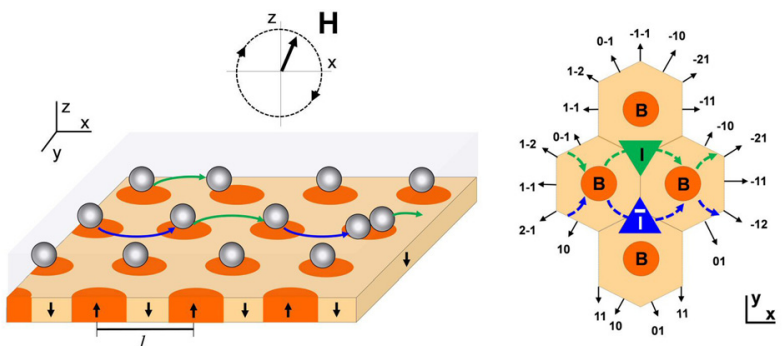

(b)

(c)

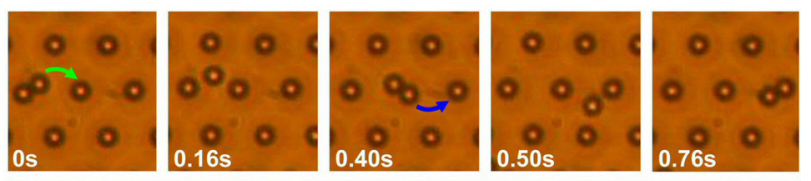

c)

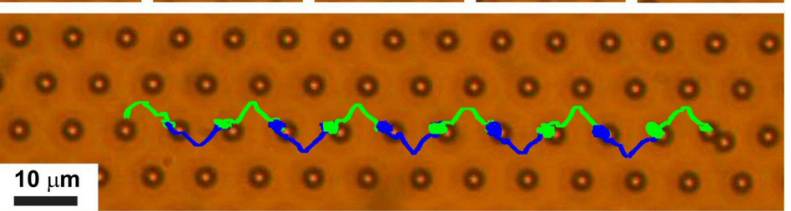

(d)
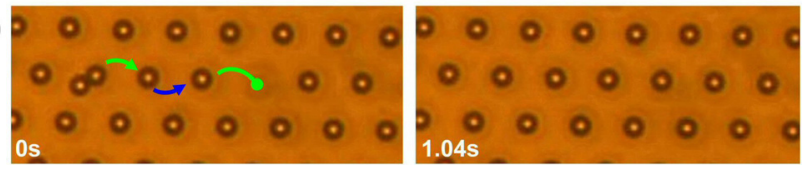

FIG. 1. (a) Sketch of the magnetic bubble lattice (lattice constant $l=8.6 \mu \mathrm{m})$ with paramagnetic colloids and subjected to a magnetic field rotating in the $(x ; z)$ plane. Schematic on the right shows four magnetic bubbles (B) with two interstitials regions $(I, \bar{I})$ illustrating the two possible particle pathways (dashed lines) and corresponding crystal directions. (b) Sequence of images showing the transport via particle swapping. (c) A particle defect transported via particle swapping, Video S1. Superimposed are the trajectories of the particles, in green (blue) are trajectories along the $B I B(B \bar{I} B)$ pathway, Video S1. (d) Filling of a lattice vacancy in the colloidal crystal, Video S2. In these experiments, the fields have amplitudes $H_{x}=0.7 \mathrm{kA} / \mathrm{m}, H_{z}=1.0 \mathrm{kA} / \mathrm{m}$ and frequency $\omega=18.8 \mathrm{~s}^{-1}$. (Multimedia view) [URL: http://dx.doi.org/10.1063/1.4874839.1] [URL: http://dx.doi.org/10.1063/1.4874839.2]

To avoid particle adhesion to the film, the FGF was coated with a $1 \mu \mathrm{m}$ thick polymer layer composed of a positive photoresist (AZ-1512, Microchem). ${ }^{20}$ Paramagnetic microspheres with $2.8 \mu \mathrm{m}$ diameter (Dynabeads M-270) were diluted in highly deionized water (milli-Q, Millipore), and deposited above the FGF. After $\sim 5$ min of sedimentation, the particles became two-dimensionally confined above the FGF due to balance between repulsive electrostatic interaction with the polymer layer, magnetic attraction and gravity.

The magnetic field applied in the $(x ; z)$ plane was generated by using two custom-made Helmholtz coils assembled above the stage of an upright microscope (Eclipse Ni, Nikon) equipped with a $100 \times 1.3 \mathrm{NA}$ objective and a $0.45 \times \mathrm{TV}$ lens. The coils were arranged perpendicular to each other and connected to two independent power amplifiers (KEPCO BOP) driven by an arbitrary waveform generator (TTi-TGA1244).

In the absence of an external field, the BWs of the magnetic bubbles attract the paramagnetic colloids and without the polymer coating, the particles sediment above the BWs. However, due to the polymer film, the particles have an higher elevation from the surface of the FGF and the magnetic potential becomes smoother, featuring energy minima at the centers of the magnetic bubbles. ${ }^{21}$ Thus, once deposited above the FGF, the particles form a perfect triangular lattice for a normalized areal density $\hat{\rho} \equiv \rho a=1$, Fig. 1. Here, $\rho=N / A$ is the particle number density, $N$ is the number of particles located within the observation area $A=140$ $\times 105 \mu \mathrm{m}^{2}$ and $a=64 \mu \mathrm{m}^{2}$ is the area of the Wigner Seitz (WS) unit cell around one bubble. For $\hat{\rho}>1$, the excess particles redistributed within the magnetic domains, and each bubble became populated by colloidal doublets, triplets, or larger clusters.

We induce particle motion by applying an external magnetic field rotating in the $(x ; z)$ plane, $\boldsymbol{H} \equiv\left(H_{x} \sin (\omega t), 0\right.$, $\left.H_{z} \cos (\omega t)\right)$, with angular frequency $\omega=2 \pi \nu$ and amplitudes $\left(H_{x} ; H_{z}\right)$, Fig. 1(a). In most of the experiments, we keep fixed the amplitude of the in-plane component $H_{x}=0.7 \mathrm{kA} / \mathrm{m}$, and change the ellipticity of the applied field by varying the amplitude of the perpendicular component, $H_{z}$.

For amplitudes $0.8 \mathrm{kA} / \mathrm{m}<H_{z}<1.2 \mathrm{kA} / \mathrm{m}$, and loading $\hat{\rho}>1$, the particle transport takes place via a swapping mechanism in doubly occupied domains, where adjacent particles synchronously exchange their positions. The bubble lattice thereby preserves the overall occupancy of one particle per domain. Increasing the particle density, the swapping motion occurs in the form of creation/destruction of doublets, triplets, or even tetramers. However, for a wide range of particle densities, we find that colloidal defects propagate mainly via doublets swapping motion, and the latter is illustrated in Fig. 1(b).

To explain the mechanism leading to the defect propagation, let us consider the arrangement of four bubbles with their interstitial regions, as shown in right part of Fig. 1(a). Energy calculations ${ }^{21}$ show that when the rotating field becomes anti-parallel with respect to the bubble magnetization, it generates in the interstitial regions two energy wells with triangular shape and opposite orientations, $I$ and $\bar{I}$, with corners pointing towards the 11 and $-1-1$ directions, respectively. In this situation, there are two equivalent pathways along which an excess particle can propagate towards the -11 direction, either along the $B \bar{I} B$ pathway (dashed blue line) or along the $B I B$ pathway (dashed green line). Both pathways are energetically equivalent, and the particle's choice is dictated by the initial orientation of the doublet in the bubble domain. A doublet initially oriented along the -21 direction will send a particle along the $B I B$ pathway (Fig. 1(b), first three images). Afterwards, this particle will form another doublet oriented towards the -12 direction, which will send a particle along the complementary $B \bar{I} B$ pathway (Fig. 1(b), last three images). When the moving particle encounters a vacancy in the colloidal lattice, it will fill it and the defect propagation will end there, as shown in Fig. 1(c).

We characterize the system conduction along the driving direction by measuring the particle flux as $J=\rho\langle v\rangle$, where $\langle v\rangle$ is the average speed as determined from particle tracking. Fig. 2(a) shows $J$ versus the dimensionless density $\hat{\rho}$, for three different frequencies. Below the loading $\hat{\rho}=1$, i.e., having less than one particle per unit cell, there are no excess particles, and thus $J=0$. For $\hat{\rho}>1$, we measure a net colloidal current which grows linearly with the loading up to $\hat{\rho} \sim 1.6$. In this regime, only the excess particles contribute to the current, while one particle per magnetic bubble does not reach the mobility threshold. The speed of the particles inside the WS unit cell is phase-locked with the driving field, and given by $v=l \omega / 2 \pi$ where $l$ is the lattice constant. Thus, 

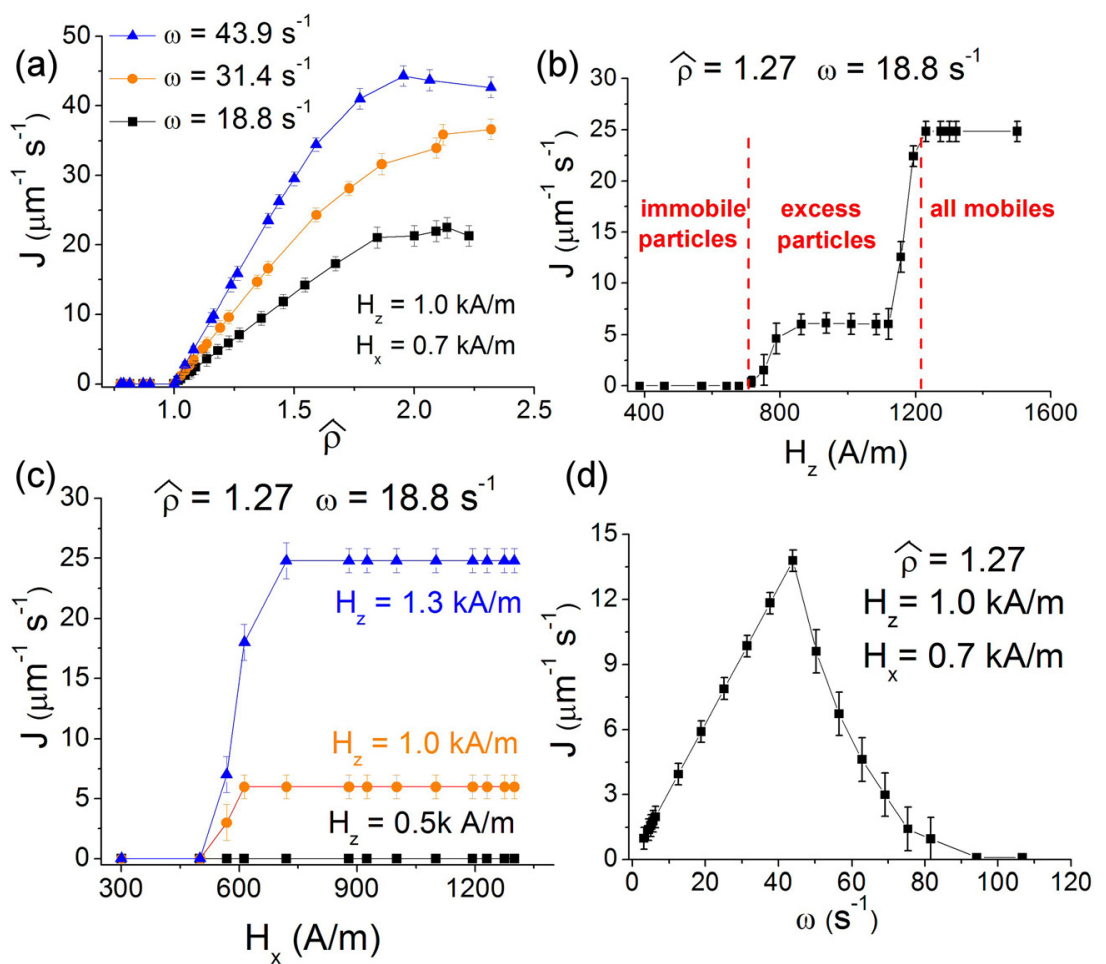

FIG. 2. (a) Particle flux $J$ versus normalized density $\hat{\rho}$ for different frequencies. (b) $J$ versus amplitude of the perpendicular field $H_{z} \quad\left(H_{x}=0.7\right.$ $\mathrm{kA} / \mathrm{m}$ ). Dashed red lines separate the regimes where all particles are immobile $\left(H_{z}<0.7 \mathrm{kA} / \mathrm{m}\right)$, only propagation of excess particles occurs $\left(0.7 \mathrm{kA} / \mathrm{m}<H_{z}<1.2 \mathrm{kA} / \mathrm{m}\right)$, and all particles are mobilized $\left(H_{z}>1.2\right.$ $\mathrm{kA} / \mathrm{m}$ ). (c) $J$ versus amplitude of the in-plane field $H_{x}$ for three different values of $H_{z}$. (d) Dependence of $J$ on the driving frequency $\omega$ for $\hat{\rho}=1.27$. increasing the driving frequency increases the average speed and, in turn, the slope of the curve in Fig. 2(a). The direction of motion of the excess particles is dictated by the chirality of the rotating field, thus, changing the polarity of one of the fields $\left(H_{x}\right.$ or $\left.H_{z}\right)$ allows to invert the entire flow of particles across the film. Increasing the loading further $(\hat{\rho}>2)$, the current reaches a maximum, and jamming between closely moving colloids forbids further particle transport.

Shown in Fig. 2(b) is the effect that the perpendicular component of the applied field $H_{z}$ has on the particle current. This component acts directly on the size of the magnetic domains, since the diameter of the magnetic bubbles increases (decreases) when $H_{z}$ is parallel (antiparallel) to the bubble magnetization. The graph, obtained for a fixed density of $\hat{\rho}=1.27$, shows that the particle flux grows in discrete steps as the amplitude of $H_{z}$ increases. Below $H_{z}=H_{1}^{c}=0.7 \mathrm{kA} / \mathrm{m}$, no current is observed. Increasing $H_{z}$, the excess particles start to be mobilized and the flux raises till reaching the constant value $J=6.1 \mu \mathrm{m}^{-1} \mathrm{~s}^{-1}$ for $H_{z}>0.7 \mathrm{kA} / \mathrm{m}$. Increasing $H_{z}$ further, reveals a second field value, $H_{2}^{c}=1.2 \mathrm{kA} / \mathrm{m}$, where the periodic displacements of the BWs are able to drive all particles synchronously across the film. The field values $H_{1}^{c}$ and $H_{2}^{c}$ are therefore the mobility edges where different sub-ensembles of particles can be set into motion.

The effect of the in-plane component of the applied field $H_{x}$ on the flux $J$ is shown in Fig. 2(c). While $H_{z}$ controls the size of the magnetic domains, the effect of $H_{x}$ is to break the spatial symmetry of the potential, inducing a net particle current towards a defined direction. When $H_{z}<H_{1}^{c}$, no current is observed for any value of $H_{x}$. For $H_{z}=1.0 \mathrm{kA} / \mathrm{m}$, the flux is composed of excess particles; while for $H_{z}=1.3 \mathrm{kA} / \mathrm{m}$, all particles are mobilized and, in both cases, when $H_{x}>$ $0.7 \mathrm{kA} / \mathrm{m}$ it becomes independent on further increasing of $H_{x}$.

Fig. 2(d) shows the dependence of $J$ on the driving frequency for $\hat{\rho}=1.27$. The current displays a full participation of the excess particles up to $\omega \sim 44 \mathrm{~s}^{-1}$, where the flux reaches its maximum value, $J=13.8 \mu \mathrm{m}^{-1} \mathrm{~s}^{-1}$ corresponding to an average defect speed of $v=60 \mu \mathrm{m} \mathrm{s}^{-1}$. Beyond this frequency, the participation of excess particles becomes partial, and the current decreases monotonously, reaching zero near $\omega=110 \mathrm{~s}^{-1}$. In this second regime, the high frequency motion of the particles is found to be intermittent, with the excess particles randomly switching between immobile and mobile phases, reducing the efficiency of our magnetic device.

Controlling the motion of the excess particles makes it possible to easily create or destroy a colloidal lattice by switching the applied field between the two mobility thresholds. This concept is demonstrated in Fig. 3 (Video S3). Starting from a triangular arrangement with one particle per WS cell (a) $(t=0 \mathrm{~s})$, we apply an elliptically polarized magnetic field with components $H_{x}=0.7 \mathrm{kA} / \mathrm{m}, H_{z}=1.3 \mathrm{kA} / \mathrm{m}>H_{2}^{c}$, and angular frequency $\omega=18.8 \mathrm{~s}^{-1}$, which mobilize the whole lattice at a speed of $25.8 \mu \mathrm{m} \mathrm{s}^{-1}$ towards the $1-1$ direction, Figs. 3(b) and 3(c), leaving the bubble substrate almost unfilled. Since the moving particles are phase-locked with the driving field for the used frequency, the translating lattice is stable and preserves the initial triangular order during motion. After $t \sim 10 \mathrm{~s}$, we change the polarity of the in-plane field $\left(H_{x} \rightarrow-H_{x}\right)$, in order to invert the particle flux towards the -11 direction. We also decrease the field amplitude to $H_{z}=0.9 \mathrm{kA} / \mathrm{m}<H_{2}^{c}$, inducing a current composed only of excess particles which start filling again the bubble lattice in the bottom part of the film, Figs. 3(d) and 3(e). Since, during these operations the top part of the film is left unfilled, we change the orientation of the applied field after $t=26.7 \mathrm{~s}$. The field now rotates in the $(y ; z)$ plane, and transports the excess particles toward the $-1-1$ direction, reforming the colloidal crystal after $31 \mathrm{~s}$. The colloidal assembly demonstrated here can be further optimized by either controlling the particle density, and thus, the amount of excess particles propagating 

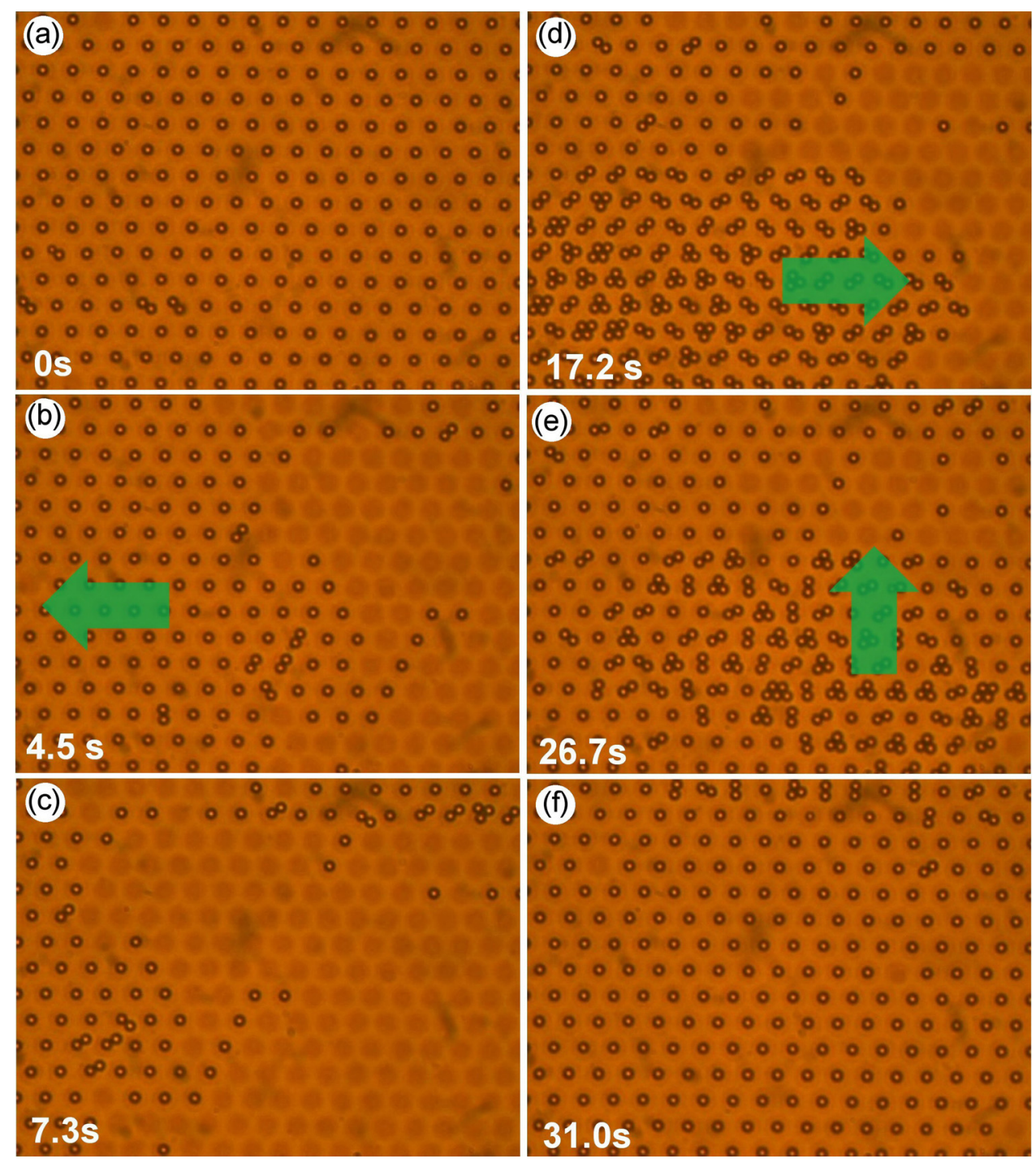

FIG. 3. (a)-(f) Sequence of images showing the reversible assembly of a colloidal lattice by switching the applied field between the two threshold fields, $H_{1}^{c}$ and $H_{2}^{c}$. First, the magnetic particles were driven toward the $1-1$ direction (green arrow in (b)) by an applied field rotating in the $(x ; z)$ plane with components $H_{x}=0.7 \mathrm{~A} / \mathrm{m}$ and $H_{z}=1.3 \mathrm{~A} / \mathrm{m}>H_{2}^{c}$, and frequency $\omega=18.8 \mathrm{~s}^{-1}$. In (d), the direction of motion is inverted and the perpendicular component of the field decreased to $H_{z}=0.9 \mathrm{kA} / \mathrm{m}<H_{2}^{c}$. In (e), the field rotates in the $(y ; z)$ plane, and the excess particles are transported towards the $-1-1$ direction, filling the whole lattice of bubbles, Video S3. (Multimedia view) [URL: http://dx.doi.org/10.1063/1.4874839.3]

through the lattice, or by increasing the driving frequency, up to a maximum speed of $v=60 \mu \mathrm{ms}^{-1}$, which will further speed up the re-writing process.

In conclusion, we demonstrate a technique to remotely generate and control the motion of defects in two dimensional lattices, while keeping track of the position of the individual particles, which could be used as model system to study the dynamics of impurities in crystalline materials. The energy scales involved in our system $\left(\sim 150 \mathrm{k}_{\mathrm{B}} \mathrm{T}\right.$, with $T \sim 293 \mathrm{~K})$ are much beyond the effect of thermal fluctuations, which could interfere with the colloidal transport process. This makes our magnetic device fully controllable, ensuring an extremely precise tuning of the particle speed and dynamics in real time and space. Although our experiments focus on using FGF films as functional platform, the technique reported here should be applicable, within the constraint of colloidal particle size and lattice wavelength, to other platforms where magnetic patterns are created by "topdown" fabrication processes.
P.T. acknowledges support from the ERC starting grant "DynaMO" (No. 335040) and from the Program Nos. RYC2011-07605 and FIS2011-15948-E. T.H.J. thanks the Research Council of Norway.

${ }^{1}$ A. Terray, J. Oakey, and D. W. M. Marr, Science 296, 1841 (2002).

${ }^{2}$ T. Sawetzki, S. Rahmouni, C. Bechinger, and D. W. M. Marr, Proc. Natl. Acad. Sci. U. S. A. 105, 20141 (2008).

${ }^{3}$ B. Kavcic, D. Babic, N. Osterman, B. Podobnik, and I. Poberaj, Appl. Phys. Lett. 95, 023504 (2009).

${ }^{4}$ U. Häfeli, W. Schutt, J. Teller, and M. Zborowsk, Scientific and Clinical Applications of Magnetic Carriers (Plenum Press, New York, London, 1997).

${ }^{5}$ M. M. Miller, P. E. Sheehan, R. L. Edelstein, C. R. Tamanaha, L. Zhong, S. Bounnak, L. J. Whitman, and R. J. Colton, J. Magn. Mater. 225, 138-144 (2001).

${ }^{6}$ D. L. Graham, H. A. Ferreira, and P. P. Freitas, Trends Biotechnol. 22, 455 (2004).

${ }^{7}$ U. Jeong, X. Teng, Y. Wang, H. Yang, and Y. Xia, Adv. Mater. 19, 33 (2007).

${ }^{8}$ K. Gunnarsson, P. E. Roy, S. Felton, J. Pihl, P. Svedlindh, S. Berner, H. Lidbaum, and S. Oscarsson, Adv. Mater. 17, 1730 (2005).

${ }^{9}$ B. Yellen, O. Hovorka, and G. Friedman, Proc. Natl. Acad. Sci. U. S. A. 102, 8860 (2005). 
${ }^{10}$ M. Donolato, P. Vavassori, M. Gobbi, M. Deryabina, M. F. Hansen, V. Metlushko, B. Ilic, M. Cantoni, D. Petti, S. Brivio, and R. Bertacco, Adv. Mater. 22, 2706 (2010).

${ }^{11}$ T. Henighan, D. Giglio, A. Chen, G. Vieira, and R. Sooryakumar, Appl. Phys. Lett. 98, 103505 (2011).

${ }^{12}$ A. Ehresmann, D. Lengemann, T. Weis, A. Albrecht, J. Langfahl-Klabes, F. Göllner, and D. Engel, Adv. Mater. 23, 5568 (2011).

${ }^{13}$ A. F. Demirörs, P. P. Pillai, B. Kowalczyk, and B. A. Grzybowski, Nature 503, 99 (2013).

${ }^{14}$ R. Seshadri and R. M. Westervelt, Phys. Rev. Lett. 70, 234 (1993).

${ }^{15}$ A. H. Eschenfelder, Magnetic Bubble Technology (Springer, Berlin Heidelberg, 1980).
${ }^{16}$ T. H. Johansen and D. V. Shantsev, Magneto-Optical Imaging, NATO Science Series II: Mathematics, Physics, and Chemistry Vol. 142 (Kluwer Academic, Dordrecht, 2004).

${ }^{17}$ P. Tierno, F. Sagués, T. H. Johansen, and T. M. Fischer, Phys. Chem. Chem. Phys. 11, 9615 (2009).

${ }^{18}$ L. E. Helseth, P. E. Goa, H. Hauglin, M. Baziljevich, and T. H. Johansen, Phys. Rev. B 65, 132514 (2002).

${ }^{19}$ P. E. Goa, H. Hauglin, A. A. F. Olsen, D. V. Shantsev, and T. H. Johansen, Appl. Phys. Lett. 82, 79 (2003).

${ }^{20}$ P. Tierno, Soft Matter 8, 11443 (2012).

${ }^{21}$ See supplementary material at http://dx.doi.org/10.1063/1.4874839 for details on the theoretical model used in the text to calculate the energy landscape. 